\title{
Beating the odds: a cardiomyocyte cell line at last
}

Jeffrey M. Leiden

Departments of Medicine and Pathology, University of Chicago, Chicago, Illinois 60637, USA

Address correspondence to: Jeffrey M. Leiden, University of Chicago, Room B608, MC 6080, 5841 S. Maryland Avenue, Chicago, Illinois 60637, USA. Phone: (773) 702-1919; Fax 773-702-1385; E-mail: jleiden@medicine.bsd.uchicago.edu

The identification of the signaling pathways and nuclear transcription factors that regulate cardiac myocyte differentiation is a problem that has both fascinated and vexed developmental biologists and cardiologists for more than 20 years. The answer to this puzzle would provide important new information about how disparate signaling pathways evolved to regulate the differentiation of closely related, but distinct, mesodermal cell lineages such as skeletal, smooth, and cardiac muscle. Of equal importance, an understanding of these pathways would have great potential clinical relevance because it might allow cardiologists to generate cultured cardiomyocyte precursors that could be used to repair damaged heart muscle in patients with a wide variety of cardiomyopathies.

Thirty years ago, most workers in the field assumed that the differentiation of a cardiac (or skeletal) muscle cell from a pluripotent mesodermal progenitor would be a hopelessly complex process, involving large numbers of growth factors, receptors, signal transduction molecules, and transcription factors. However, a pioneering study of skeletal muscle differentiation by Jones and colleagues (1) in the 1970s turned this notion on its head. In an elegant series of experiments, they showed, much to our surprise, that cultured $10 \mathrm{~T} 1 / 2$ fibroblasts could be driven to differentiate into skeletal muscle by altering the expression of a very small number of genes by treatment of the cells with the DNA demethylating agent 5-azacytidine. Following up on this initial observation, Lassar, Weintraub et al. (2) subsequently identified a novel gene called MyoD that when transfected alone into 10T1/2 fibroblasts could convert them into skeletal myoblasts. Subsequent studies demonstrated that MyoD was a basic helix-loop-helix transcription factor that belonged to a family of four related myogenic determining genes. Binding sites for these myogenic transcription factors were identified in the promoters of most, if not all, skeletal muscle-specific genes, and overexpression of any of the four MyoD-like factors alone could convert cultured fibroblasts to skeletal myocytes in vitro. Conversely, mice deficient in one or more of these genes demonstrated severe defects in skeletal muscle differentiation (reviewed in ref. 3 ).

By analogy with the skeletal muscle lineage, it has been assumed that the differentiation of cardiac muscle from lateral plate mesodermal precursors is also controlled by a small number of signaling pathways and transcription factors. However, progress in identifying and characterizing these pathways has been slowed immeasurably by the lack of a cultured progenitor cell line that could be differentiated at will into committed cardiomyocytes in vitro. In this issue of

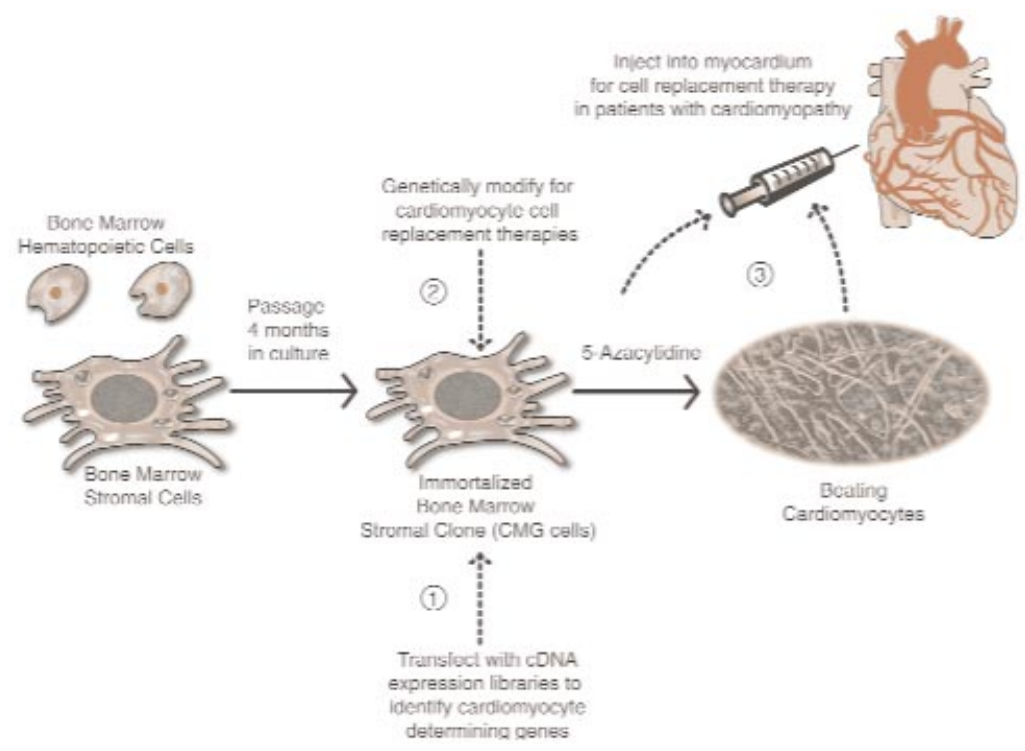

Figure 1

Derivation and potential applications of bone marrow stroma-derived cardiomyocyte cell lines. Schematic illustration of the derivation of the CMG bone marrow stroma-derived cardiomyocyte cell line. Solid arrows indicate the work reported by Ogawa and colleagues (4) in this issue of the JCI. Dotted lines show potential applications of the cardiomyocyte cell line in studies designed to identify cardiomyocyte-determining genes (1), or in cell replacement therapies for patients with cardiomyopathy $(2,3)$ 
the JCI, Ogawa and colleagues (4) appear to have finally solved this problem and to have done so in an innovative and surprising manner.

Recent studies from several groups have suggested that bone marrow stromal (BMS) cells display several characteristics of a pluripotent mesenchymal stem cell. Such BMS cells, for example, can differentiate into multiple cell lineages, including bone, muscle, and fat. Ogawa and colleagues therefore hypothesized that these same cells might also have the potential to differentiate into cardiomyocyte precursors. Indeed, after immortalizing BMS cells by prolonged culture in vitro, they were able to identify a single clone of adherent fibroblastlike cells that when treated with 5 -azacytidine would reproducibly differentiate into beating myocytes that displayed many properties of embryonic ventricular muscle. After differentiation, these cells, which they called CMG (cardiomyogenic), acquired many morphologic features of cardiac muscle, including sarcomeres, one to three centrally located nuclei, and atrial granules (which are also seen in embryonic ventricular cardiomyocytes). They also expressed several cardiac-specific genes, including the GATA4 and Nkx2.5 transcription factors and the brain nateiuretic peptide (BNP) and atrial nateiuretic factor (ANF) genes. In addition, they displayed cardiac-like action potentials with a shallow resting membrane potential, a long action potential duration, and a late diastolic slow depolarization current.

The identification and initial characterization of the CMG cell line will be of great interest to both cardiovascular biologists and clinicians. The finding that adult bone marrow stromal cells have the capacity to give rise to cardiomyocytes identifies, for the first time, a readily available (and until now unap- preciated) source of precursors for both basic and clinical studies of cardiomyocyte development and function. The fact that the CMG cells can be efficiently differentiated into cardiomyocytes after treatment with 5-azacytidine suggests that cardiac myocyte differentiation, like skeletal muscle development, is controlled by a very small number of genes and signaling pathways. Moreover, the CMG cells represent an ideal model system for transfection experiments designed to identify these cardiac muscle-determining genes. In this regard, it is of interest that before differentiating into cardiomyocytes, the CMG cells express GATA4, Nkx2.5, and TEF1, three transcription factors that have been implicated in early cardiomyocyte differentiation (reviewed in ref. 5). This finding suggests that transfection experiments in CMG cells may lead to the identification of novel transcription factors that regulate the differentiation of the cardiac muscle cell lineage.

The availability of transfectable cultured cardiomyocytes will also facilitate basic studies of cardiac promoters and structure-function studies of cardiomyocyte proteins that, until now, required the isolation and transfection of primary neonatal cardiomyocytes. Finally, a recent study from several groups (6) has suggested that embryonic cardiomyocytes can be injected into the adult ventricular myocardium where they are stably incorporated into cardiac muscle, thus raising the possibility of using cell replacement therapies to treat a wide variety of cardiomyopathies. The finding by Ogawa and colleagues that bone marrow stromal cells can be used to generate embryonic cardiac muscle suggests the possibility of using these cultured bone marrow cells to generate new cardiac muscle for such cell-based therapies. Moreover, such cultured procardiomyocytes will likely be amenable to genetic manipulation, which could serve as a useful adjunct to cell-based therapies in patients with cardiomyopathies.

As is true of all exciting new discoveries, the work of Ogawa and colleagues also raises a number of important questions. What is the identity of the bone marrow stromal cell that can give rise to cardiomyocytes, and how many of these cells exist in the normal adult marrow? How easy and reproducible is the isolation of CMG-like clones from rodent bone marrow, and can such cells also be isolated from human marrow? Are there specific growth conditions that promote the proliferation, survival, and differentiation of the cardiomyocyte precursor? Are the CMG cells irreversibly committed to the cardiomyocyte lineage, or can they also give rise to other mesodermal cell types? And finally, can cardiomyocytes derived from the CMG cells be stably incorporated into adult ventricular muscle after direct intramyocardial injection. Given the excitement surrounding the isolation of the CMG cells, it is safe to predict that we won't have long to wait for the answers to many of these questions and for the use of these cells in a variety of novel basic and clinical studies of heart development.

\footnotetext{
1. Taylor, S.M., and Jones, P.A. 1979. Multiple new phenotypes induced in $10 \mathrm{~T} 1 / 2$ and $3 \mathrm{~T} 3$ cells treated with 5-azacytidine. Cell. 4:771-779.

2. Davis, R.L., Weintraub, H., and Lassar, A.B. 1987. Expression of a single transfected cDNA converts fibroblasts to myoblasts. Cell. 6:987-1000.

3. Olson, E.N., and Klein, W.H. 1994. bHLH factors in muscle development: dead lines and commitments, what to leave in and what to leave out Genes Dev. 8:1-8.

4. Makino, S., et al. 1999. Cardiomyocytes can be generated from marrow stromal cells in vitro. $J$. Clin. Invest. 103:697-705.

5. Fishman, M.C., and Chien, K.R. 1997. Fashioning the vertebrate heart: earliest embryonic decisions. Development. 124:2099-2117.

6. Soonpaa, M.H., Koh, G.Y, Klug, M.G., and Field, L.J. 1994. Formation of nascent intercalated disks between grafted fetal cardiomyocytes and host myocardium. Science. 264:98-101.
} 